\title{
Fluorescence Assessment of the AmpR-Signaling Network of Pseudomonas aeruginosa to Exposure to $\beta$-Lactam Antibiotics
}

\author{
David A. Dik, ${ }^{\dagger, \#, \|}$ Choon Kim, ${ }^{\dagger, \#}$ Chinedu S. Madukoma,${ }^{\ddagger}$ Jed F. Fisher, ${ }^{\dagger}$ \\ Joshua D. Shrout, \\ ${ }^{\dagger}$ Department of Chemistry \& Biochemistry, University of Notre Dame, Notre Dame, Indiana 46556, United States \\ *Department of Civil \& Environmental Engineering \& Earth Sciences, University of Notre Dame, Notre Dame, \\ Indiana 46556, United States \\ $\S$ Department of Biological Sciences, University of Notre Dame, Notre Dame, IN 46556, United States \\ ${ }^{\#}$ D.A.D. and C.K. contributed equally to this work. \\ * To whom correspondence should be addressed. Email: mobashery@,nd.edu
}

Table of Contents

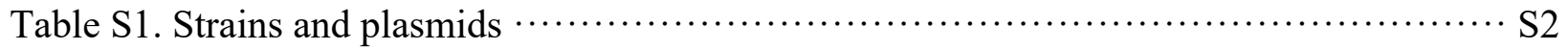

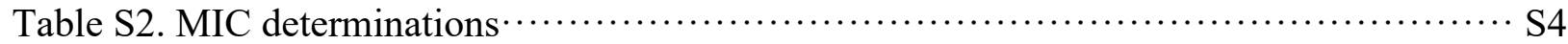

Table S3. MIC determinations for additional antibiotics, non- $\beta$-lactams included $\cdots \cdots \cdots \cdots \cdot$. S5

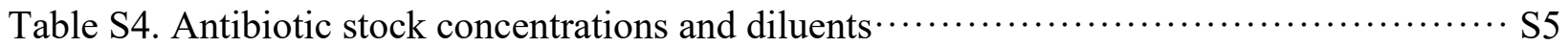

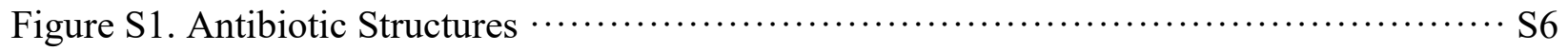

Figure S2. Fluorescence assay for wild-type $P$. aeruginos $a$ with $\beta$-lactam antibiotics $\cdots \cdots \cdots \cdot$. S7

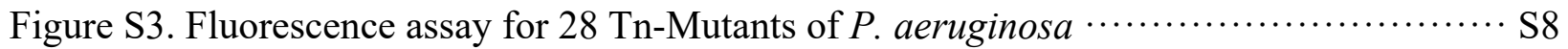

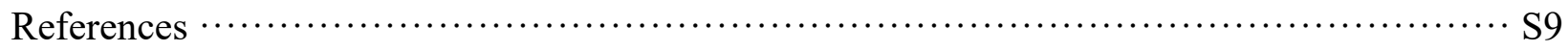


Table S1: Bacterial strains and plasmids.

\begin{tabular}{|c|c|c|}
\hline Strain or plasmid & Description & Reference \\
\hline \multicolumn{3}{|l|}{ P. aeruginosa } \\
\hline $\begin{array}{l}\text { Wild-type (MPAO1) } \\
\text { Wild-type (MPAO1) } \\
\text { Wild-type (MPAO1) } \\
\text { Wild-type (MPAO1) }\end{array}$ & $\begin{array}{l}\text { Wild-type:: pCN61_AmpRtdT, AMPr', STR } \\
\text { Wild-type:: pCN61_AmpRCtdT, AMPr', STR } \\
\text { Wild-type:: pCN61_AmpRgfp, AMP }{ }^{r} \text {, STR } \\
\text { Wild-type:: pCN61_AmpRCgfp, AMP }{ }^{r} \text {, STR }\end{array}$ & $\begin{array}{l}\text { This study } \\
\text { This study } \\
\text { This study } \\
\text { This study }\end{array}$ \\
\hline \multicolumn{3}{|l|}{$\begin{array}{l}\text { P. aeruginosa } \\
\text { Transposon-insertion mutants }\end{array}$} \\
\hline 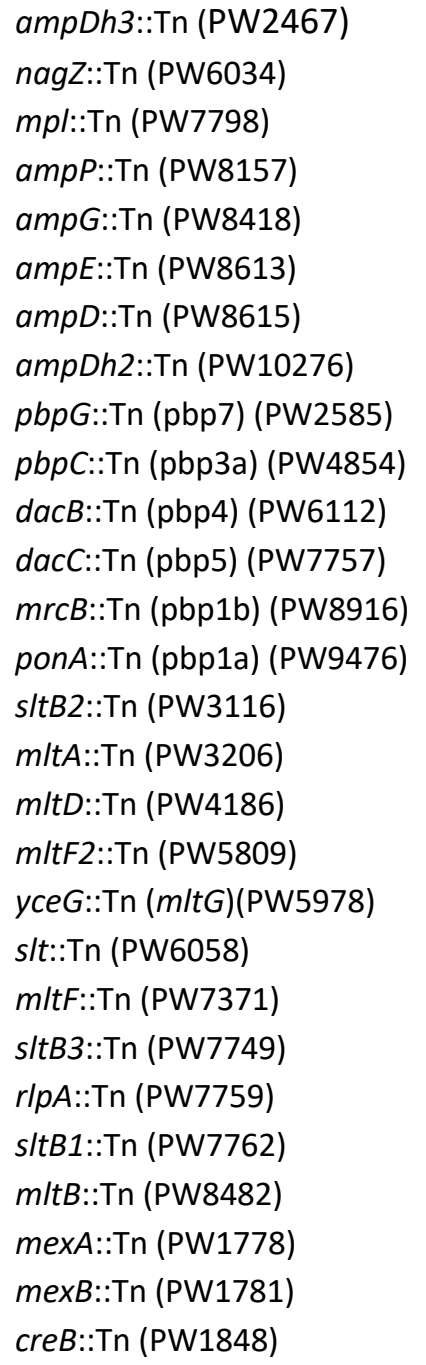 & 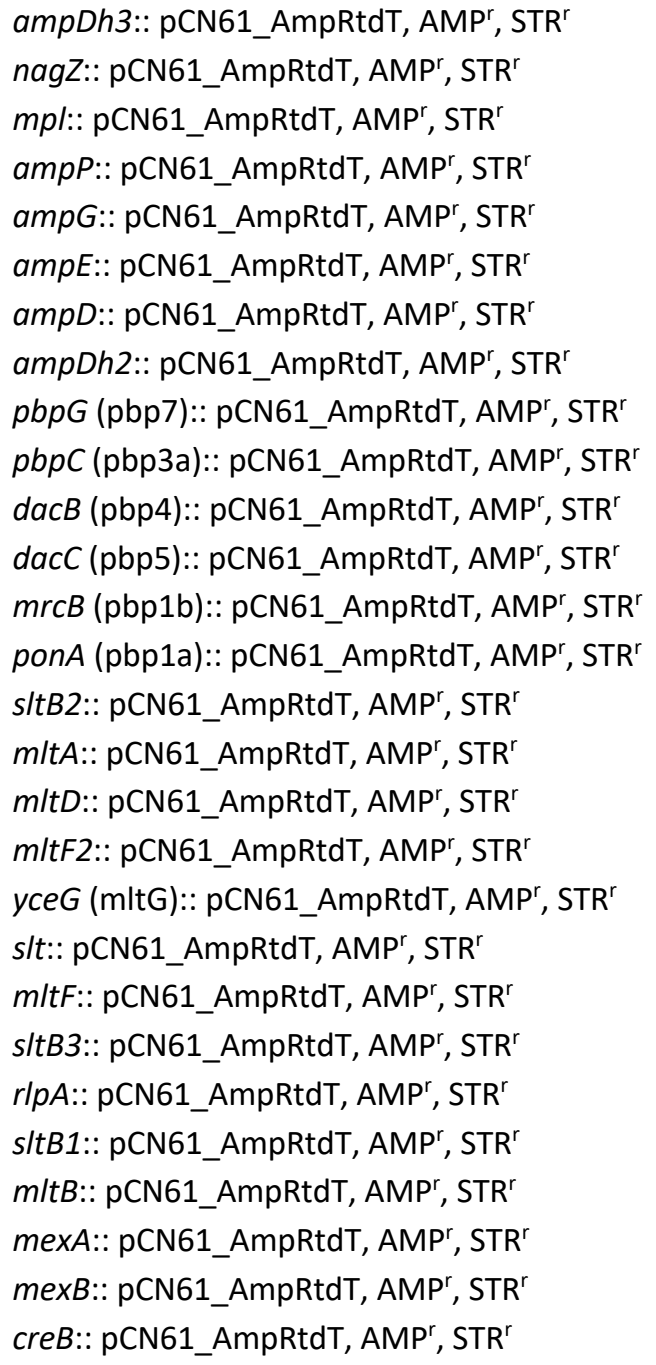 & $\begin{array}{l}\text { This study } \\
\text { This study } \\
\text { This study } \\
\text { This study } \\
\text { This study } \\
\text { This study } \\
\text { This study } \\
\text { This study } \\
\text { This study } \\
\text { This study } \\
\text { This study } \\
\text { This study } \\
\text { This study } \\
\text { This study } \\
\text { This study } \\
\text { This study } \\
\text { This study } \\
\text { This study } \\
\text { This study } \\
\text { This study } \\
\text { This study } \\
\text { This study } \\
\text { This study } \\
\text { This study } \\
\text { This study } \\
\text { This study } \\
\text { This study } \\
\text { This study }\end{array}$ \\
\hline
\end{tabular}


Table S1 (Cont.)

\begin{tabular}{|c|c|c|}
\hline Strain or plasmid & Description & Reference \\
\hline \multicolumn{3}{|c|}{ GFP-labeled $P$. aeruginosa } \\
\hline $\begin{array}{l}\text { Wild-type (PAO1C-gfp) } \\
\text { Wild-type (PAO1C-gfp) } \\
\text { (pCN61_AmpRtdT) }\end{array}$ & $\begin{array}{l}\text { Wild-type:: miniTn7-gfp2, } \mathrm{CHL}^{r} \text {, AMPr }{ }^{r} \text {, GEN } \\
\text { Wild-type:: miniTn7-gfp2, } \mathrm{CHL}^{r} \text {, AMPr }{ }^{r}, \mathrm{GEN}^{\mathrm{r}} \text {; } \\
\text { pCN61_AmpRtdT, AMPr, STR }\end{array}$ & $\begin{array}{l}\text { (Madukoma } \\
\text { et al. 2019) } \\
\text { This study }\end{array}$ \\
\hline \multicolumn{3}{|l|}{ Other Strains } \\
\hline P. mesacidophila & (ATCC 31433) & \\
\hline B. licheniformis & (NRS 712) & \\
\hline M. xanthus & (DK1622) & \\
\hline E. coli & (ATCC 10798) & \\
\hline \multicolumn{3}{|l|}{ Plasmids } \\
\hline pCN61_AmpRtdT & pCN61_AmpR_tdTomato, AMPr ${ }^{r}, S^{r}{ }^{r}$ & This study \\
\hline pCN61_AmpRCtdT & pCN61_AmpR_AmpC_tdTomato, AMPr',STR & This study \\
\hline pCN61_AmpRgfp & pCN61_AmpR_GFP, AMPr, STR $^{r}$ & This study \\
\hline pCN61_AmpRCgfp & pCN61_AmpR_AmpC_GFP, AMPr ${ }^{r}$ STR $^{r}$ & This study \\
\hline AKN66 & mini-Tn7-gfp2; CHL' ${ }^{r}, E^{r}$ & $\begin{array}{l}\text { (Koch et al. } \\
2001)^{2}\end{array}$ \\
\hline pUX-BF13 & Conjugation helper plasmid, $\mathrm{AMP}^{r}$ & $\begin{array}{l}\text { (Bao et al. } \\
1991)^{3}\end{array}$ \\
\hline pRK600 & Mobilization plasmid, $\mathrm{CHL}^{\mathrm{r}}$ & $\begin{array}{l}\text { (Kessler et } \\
\text { al. 1992) }\end{array}$ \\
\hline
\end{tabular}

AMP, ampicillin; CHL, chloramphenicol; GEN, gentamycin; STR, streptomycin 
Table S2: Susceptibility of Tn mutants to $\beta$-lactam antibiotics inducing ampC expression.

\begin{tabular}{|c|c|c|c|c|}
\hline \multirow{2}{*}{ Strain } & \multicolumn{4}{|c|}{$\mathrm{MIC}(\mu \mathrm{g} / \mathrm{ml})^{\mathrm{a}}$} \\
\hline & CAZ & FOX & IPM & MEM \\
\hline \multicolumn{5}{|l|}{ Wild type } \\
\hline $\mathrm{MPAO}^{\mathrm{b}}$ & 0.5 & 1024 & 1 & 0.5 \\
\hline \multicolumn{5}{|l|}{$\begin{array}{l}\text { Tn mutant } \\
\text { Lytic transglycosylase }\end{array}$} \\
\hline PW3116 (sltB2) & 0.5 & 1024 & 1 & 1 \\
\hline PW3206 (m/tA) & 1 & 1024 & 1 & 1 \\
\hline PW4186 (m/tD) & 1 & 1024 & 1 & 0.5 \\
\hline PW5809 (m/tF2) & 0.5 & 1024 & 1 & 0.5 \\
\hline PW5978 (yceG, mltG) & 1 & 512 & 0.25 & 0.5 \\
\hline PW6058 (s/t) & 0.5 & 1024 & 1 & 0.5 \\
\hline PW7371 (m/tF) & 1 & 10.24 & 1 & 0.5 \\
\hline PW7749 (s/tB3) & 0.5 & 1024 & 1 & 0.5 \\
\hline PW7759 (rlpA) & 1 & 1024 & 1 & 0.5 \\
\hline PW7762 (s/tB1) & 0.5 & 1024 & 1 & 1 \\
\hline \multicolumn{5}{|l|}{ Peptidoglycan recycling } \\
\hline PW2467 (ampDh3) & 1 & 1024 & 4 & 2 \\
\hline PW6034 (nagZ) & 1 & 1024 & 1 & 0.5 \\
\hline PW7798 (mpl) & 1 & 1024 & 0.5 & 1 \\
\hline PW8157 (ampP) & 1 & 1024 & 1 & 0.5 \\
\hline PW8418 (ampG) & 1 & 1024 & 1 & 0.5 \\
\hline PW8613 (ampE) & 1 & 1024 & 1 & 0.5 \\
\hline PW8615 (ampD) & 8 & 1024 & 1 & 4 \\
\hline \multicolumn{5}{|c|}{$\underline{\text { Penicillin-binding proteins }}$} \\
\hline PW2585 (pbpG, pbp7) & 1 & 1024 & 1 & 0.5 \\
\hline PW4854 (pbpC, pbp3a) & 1 & 1024 & 1 & 0.5 \\
\hline PW6112 (dacB, pbp4) & 16 & 1024 & 1 & 1 \\
\hline PW7757 (dacC, pbp5) & 1 & 1024 & 1 & 0.5 \\
\hline PW8916 (mrcB, pbp1b) & 1 & 1024 & 1 & 0.5 \\
\hline PW9476 (ponA, pbp1a) & 1 & 512 & 0.5 & 1 \\
\hline \multicolumn{5}{|c|}{ Efflux/Two-component system } \\
\hline PW1778 (mexA) & 0.5 & 512 & 1 & 0.25 \\
\hline PW1781 (mеxB) & 0.5 & 512 & 1 & 0.25 \\
\hline PW1848 (creB) & 1 & 1024 & 1 & 0.5 \\
\hline
\end{tabular}


Table S3: Susceptibility of Tn mutants to additional antibiotics including non- $\beta$-lactam drugs.

\begin{tabular}{|c|c|c|c|c|c|c|}
\hline \multirow{2}{*}{ Strain } & \multicolumn{6}{|c|}{$\mathrm{MIC}(\mu \mathrm{g} / \mathrm{ml})^{\mathrm{a}}$} \\
\hline & ATM & CFS & MEC & $\mathrm{FOF}^{\mathrm{b}}$ & GEN $^{b}$ & $\mathrm{TOB}^{\mathrm{b}}$ \\
\hline \multicolumn{7}{|l|}{ Wild type } \\
\hline MPAO1 & 1 & 1 & 256 & 128 & 0.5 & 0.25 \\
\hline \multicolumn{7}{|l|}{ Tn mutant } \\
\hline PW8615 (ampD) & 8 & 4 & 512 & 64 & 2 & 0.5 \\
\hline PW8418 (ampG) & 4 & 2 & 256 & 128 & 1 & 0.5 \\
\hline PW4186 (m/tD) & 4 & 2 & 256 & 64 & 1 & 0.5 \\
\hline PW7371 (m/tF) & 4 & 1 & 256 & 64 & 1 & 0.5 \\
\hline PW6034 (nagZ) & 4 & 32 & 1024 & 128 & 1 & 0.25 \\
\hline PW6112 (dacB, pbp4) & 16 & 16 & 256 & 64 & 1 & 0.25 \\
\hline PW6058 (s/t) & 2 & 1 & 128 & 64 & 1 & 0.25 \\
\hline PW5978 (yceG, mltG) & 4 & 2 & 64 & 64 & 1 & 0.25 \\
\hline
\end{tabular}

${ }^{a}$ ATM, aztreonam; CFS, cefsulodin; MEC, mecillinam; FOF, fosfomycin;

GEN, gentamicin; TOB, tobramycin. ${ }^{b}$ Non- $\beta$-lactam antibiotics.

Table S4: Susceptibility of Tn mutants to additional antibiotics including non- $\beta$-lactam drugs.

\begin{tabular}{l|l} 
Antibiotic & Conc. and Diluent \\
\hline CAZ & $10 \mathrm{mg} / \mathrm{ml}$ in DMSO \\
MEM & $50 \mathrm{mg} / \mathrm{ml}$ in DMSO \\
IPM & $4 \mathrm{mg} / \mathrm{ml}$ in $\mathrm{H}_{2} \mathrm{O}$ \\
ATM & $30 \mathrm{mg} / \mathrm{ml}$ in DMSO \\
CFS & $50 \mathrm{mg} / \mathrm{ml}$ in $\mathrm{H}_{2} \mathrm{O}$ \\
MEC & $5 \mathrm{mg} / \mathrm{ml}$ in $\mathrm{MHII}$ \\
FOF & $25 \mathrm{mg} / \mathrm{ml}$ in $\mathrm{H}_{2} \mathrm{O}$ \\
GEN & $50 \mathrm{mg} / \mathrm{ml}$ in $\mathrm{H}_{2} \mathrm{O}$ \\
TOB & $50 \mathrm{mg} / \mathrm{ml}$ in $\mathrm{H}_{2} \mathrm{O}$ \\
FOX & $50 \mathrm{mg} / \mathrm{ml}$ in $\mathrm{H}_{2} \mathrm{O}$
\end{tabular}


A

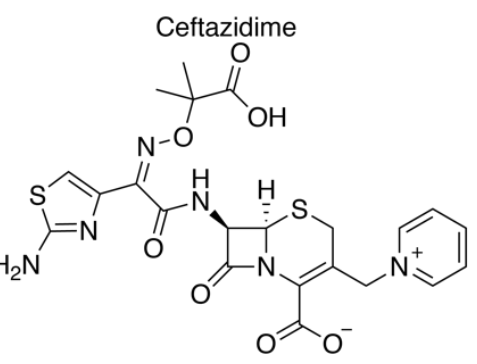

C<smiles>CC(O)[C@H]1C(=O)N2C(C(=O)[O-])=C(SCCNC=[NH2+])C[C@H]12</smiles>

E<smiles>NC(=O)c1cc[n+](CC2=C(C(=O)[O-])N3C(=O)[C@@H](NC(=O)[C@H](c4ccccc4)S(=O)(=O)O)[C@H]3SC2)cc1</smiles>

G

Fosfomycin<smiles>CC1OC1P(=O)(O)O</smiles>

I

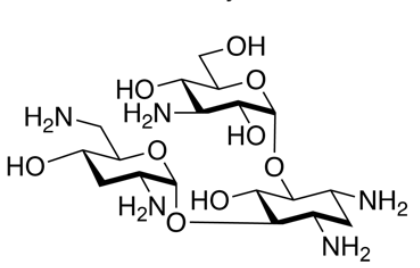

B

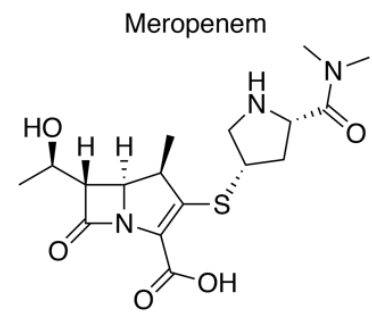

D

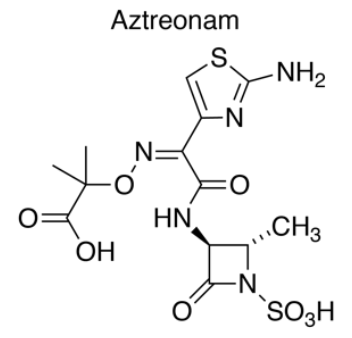

$\mathbf{F}$

Mecillinam<smiles>CC1(C)S[C@@H]2[C@H](N=CN3CCCCCC3)C(=O)N2[C@H]1C(=O)O</smiles>

H Gentamicin $\mathrm{C}_{2}$

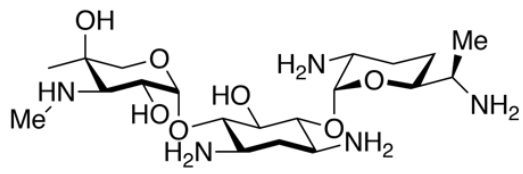

$\mathbf{J}$

Cefoxitin<smiles>CO[C@]1(NC(=O)Cc2cccs2)C(=O)N2C(C(=O)[O-])=C(COC(N)=O)CS[C@H]21</smiles>

Figure S1. The structure of each antibiotic is given: (A) ceftazidime (CAZ), (B) meropenem (MEM), (C) imipenem (IPM), (D) aztreonam (ATM), (E) cefsulodin (CFS), (F) mecillinam (MEC), (G) fosfomycin (FOF), (H) gentamicin $\mathrm{C}_{2}(\mathrm{GEN})$, (I) tobramycin (TOB), (J) cefoxitin (FOX). Gentamicin is a mixture of three compounds $\left(\mathrm{C}_{1}, \mathrm{C}_{1 \mathrm{~A}}, \mathrm{C}_{2}\right)$, which are distinguished by the methylation at $\mathrm{C} 6$ of the saccharide bonded to $\mathrm{C} 4$ of the 2-deoxystreptamine. ${ }^{5}$ The $\mathrm{C}_{2}$ structure of gentamicin is shown. 


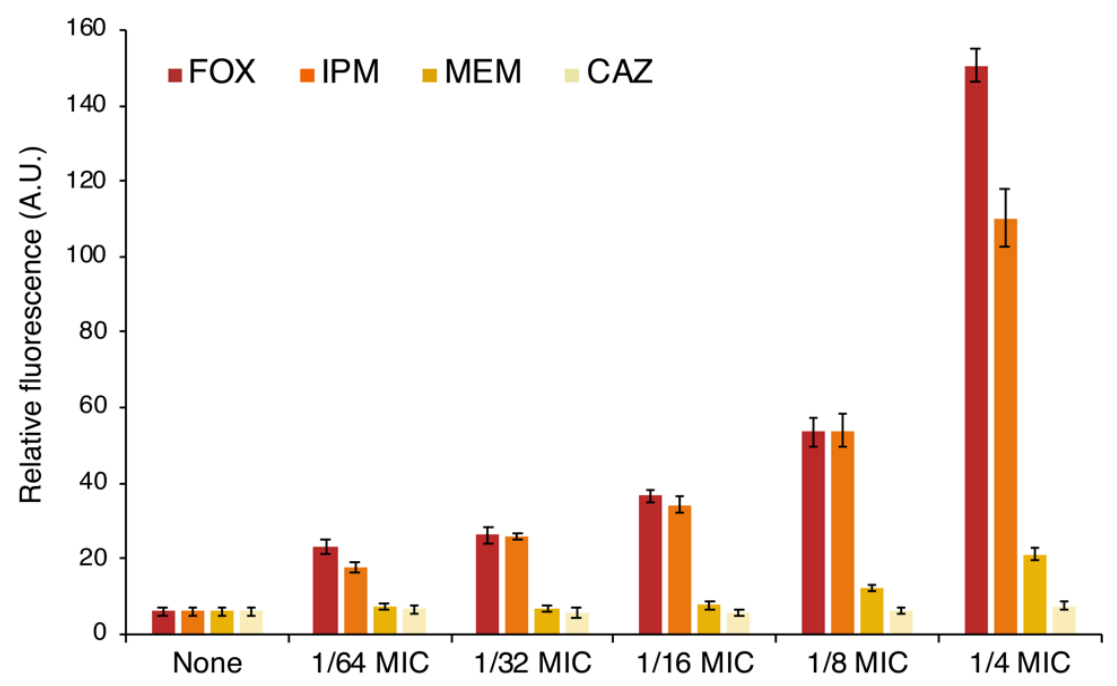

Figure S2. The relative fluorescence intensity (A.U.) of tdTomato (representative of AmpC $\beta$ lactamase production) for wild-type $P$. aeruginosa PAO1 on exposure of the bacteria to no antibiotic or $1 / 64,1 / 16,1 / 8$ and $1 / 4$ MIC concentrations of FOX (red), IPM (orange), MEM (gold), and CAZ (beige). The experiments were performed on parafilm in triplicate. The error bars show the standard deviation of the measurements. FOX and IPM are more potent inducers of $a m p C$ than are MEM or CAZ in wild-type $P$. aeruginosa. 

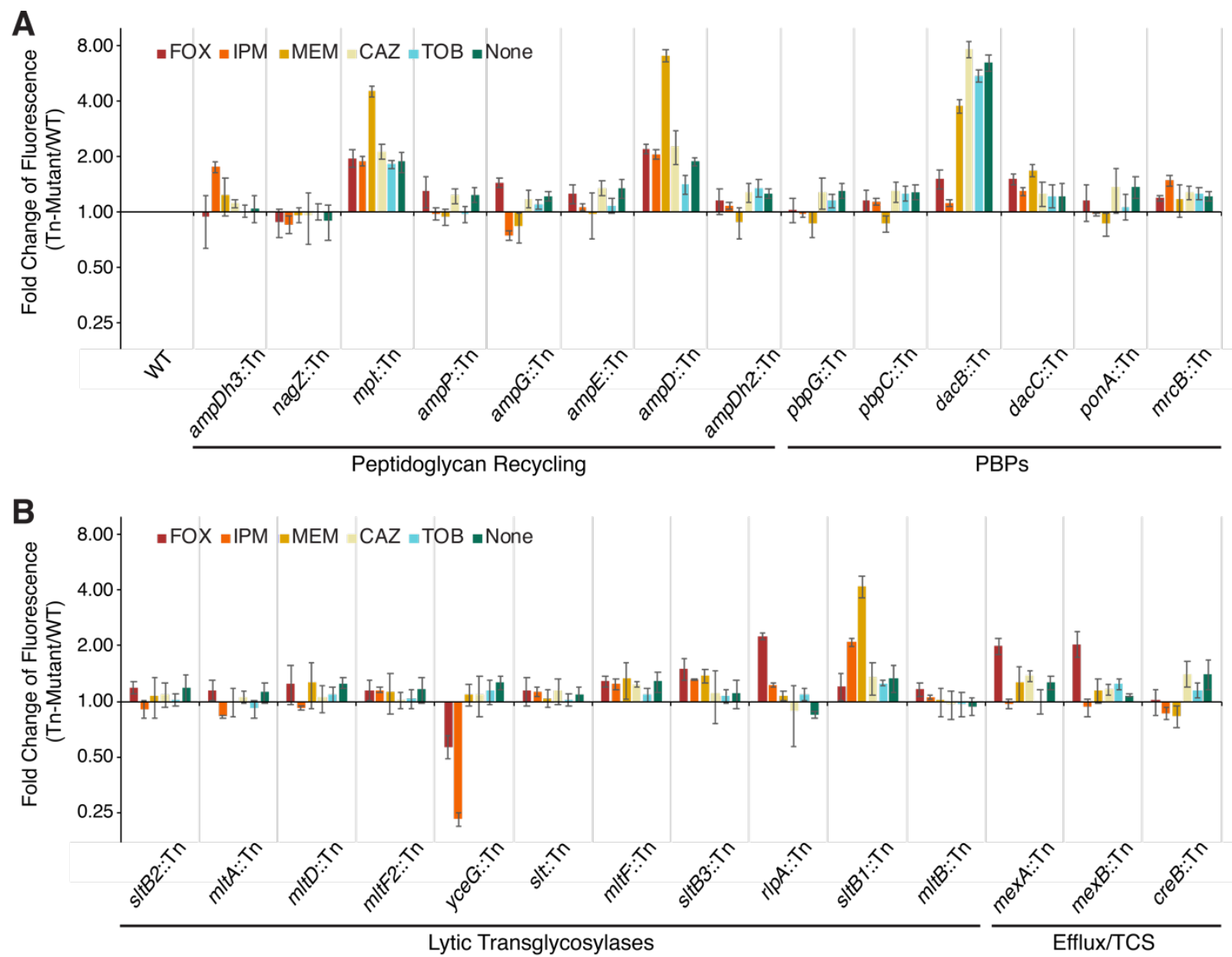

Figure S3. The fold-change of the fluorescence intensity of tdTomato, corresponding to AmpC $\beta$ lactamase production, for transposon-mutant strains on exposure of the bacteria to $1 / 8$ MIC concentrations of FOX (red), IPM (orange), MEM (gold), CAZ (beige), TOB (blue) and no antibiotic (turquoise) is shown. (A) We screened transposon-insertion mutant strains for enzymes implicated in peptidoglycan recycling and the PBPs. (B) Additionally, we screened transposoninsertion mutant strains for the lytic transglycosylases, enzymes implicated in antibiotic efflux, and $\mathrm{CreB}$ a two component-regulator resistance determinant. The experiments were performed in triplicate and normalized to the fluorescence intensity measured for the wild-type strain. The error bars give the standard deviation of the three data points. 


\section{References}

(1) Madukoma, C. S., Liang, P., Dimkovikj, A., Chen, J., Lee, S. W., Chen, D. Z., Shrout, J. D. (2019) Single cells exhibit differing behavioral phases during early stages of Pseudomonas aeruginosa swarming. J. Bacteriol. 201, e00184-19.

(2) Koch, B., Jensen, L. E., Nybroe, O. (2001) A panel of Tn7-based vectors for insertion of the gfp marker gene or for delivery of cloned DNA into Gram-negative bacteria at a neutral chromosomal site. J. Microbiol. Methods 45, 187-195.

(3) Bao, Y., Lies, D. P., Fu, H., Roberts, G. P. (1991) An improved Tn7-based system for the single-copy insertion of cloned genes into chromosomes of Gram-negative bacteria. Gene 109, 167-168.

(4) Kessler, B., de Lorenzo, V., Timmis, K. N. (1992) A general system to integrate lacZ fusions into the chromosomes of Gram-negative eubacteria: Regulation of the Pm promoter of the TOL plasmid studied with all controlling elements in monocopy. Mol. Gen. Genet. 233, 293-301.

(5) Park, J. W., Hong, J. S., Parajuli, N., Jung, W. S., Park, S. R., Lim, S. K., Sohng, J. K., Yoon, Y. J. (2008) Genetic dissection of the biosynthetic route to gentamicin A2 by heterologous expression of its minimal gene set. Proc. Natl. Acad. Sci. U. S. A. 105, 83998404. 